\title{
Percepción de satisfacción y calidad de servicios odontológicos en una clínica universitaria de Cartagena, Colombia
}

Irene Margarita Lora-Salgado, Esp. . , Lesbia Rosa Tirado-Amador ${ }^{*}, \mathrm{OD}_{2}$, Jorge Luis Montoya-Mendoza, Esp. ${ }_{1}$, Miguel Ángel Simancas-Pallares, $\mathrm{OD}_{2}$

${ }_{1}$ Universidad del Sinú, seccional Cartagena, Bolívar, Colombia

${ }_{2}$ Universidad de Cartagena, Cartagena, Bolivar, Colombia

Recibido: 28 de febrero del 2016 Aprobado: 16 de marzo del 2016

*Autor de correspondencia: Lesbia Rosa Tirado Amador. Avenida Pedro de Heredia n. ${ }^{\circ}$ 31-176 sector Amberes, Cartagena, Colombia. Correo electrónico: investigacionesodontologia@unisinucartagena.edu.co

Cómo citar este artículo: Lora-Salgado IM, Tirado-Amador LR, Montoya-Mendoza JL, Simancas-Pallares MÁ. Percepción de satisfacción y calidad de servicios odontológicos en una clínica universitaria de Cartagena, Colombia. Rev Nac Odontol. 2016;12(23):31-40. doi: 10.16925/od.v12i23.1378

Resumen. Introducción: el objetivo de esta investigación fue describir el nivel de calidad y satisfacción percibido por usuarios de los servicios odontológicos de una clínica universitaria en Cartagena, Colombia. Métodos: estudio de corte transversal en 277 pacientes. Para la recolección de la información, se hizo una encuesta estructurada autosuministrada que contenía variables demográficas, información asociada a la prestación del servicio y preguntas específicas para calidad y satisfacción en los servicios. El análisis de la información se llevó a cabo con estadística descriptiva a partir de frecuencias y porcentajes, intervalos de confianza al $95 \%$ y análisis bivariado en el que se utilizó la prueba Chi-cuadrado para establecer asociación entre las variables. Resultados: más de la mitad de los usuarios de los servicios odontológicos fueron mujeres; la edad promedio de los participantes fue de 39 años (DE: 12,48); 99,3\% (IC 95\%: 98,2-100) de los sujetos manifestó estar satisfecho con los servicios, y 97,8\% (IC 95\%: 96,1 99,5) percibió que la atención se presta con calidad. Al relacionar las variables sociodemográficas con el nivel de calidad y satisfacción percibido, no se encontró asociación estadísticamente significativa con ninguna de las variables estudiadas. Conclusión: el nivel de calidad y la satisfacción global en los servicios odontológicos se perciben satisfactoriamente por los usuarios; sin embargo, esto no es evidencia fehaciente de la existencia de tal calidad, lo que obliga a la institución a hacer seguimiento de la satisfacción de los usuarios, dado que en algunos indicadores como el recurso humano existe evidencia de inconformidad.

Palabras clave: atención odontológica, calidad de la atención de salud, relaciones médico-paciente, satisfacción del paciente. 


\title{
Satisfaction and Quality Perception of odontology services in a University Clinic of Cartagena, Colombia
}

\begin{abstract}
Introduction: The objective of this research is to describe the quality and satisfaction levels perceived by users of the odontology services in a university clinic in Cartagena, Colombia. Methods: Cross-sectional study in 277 patients. In order to collect information, a self-supplied structured survey was given to the patients that included demographic variables, information associated to the service delivered and specific questions for the quality and satisfaction of the services. The information analysis was performed with descriptive statistics from frequencies and percentages, confidence intervals to $95 \%$ and bivariate analysis where the Chi-square test was used to determine the association amongst variables. Results: More than half of the dental service users were women, the average age of the participants was 39 (from: 12.48) years old; 99.3\% (ic 95\%: 98.2-100) of the participants indicated service satisfaction, and 97.8\% (ic 955: 96.1-99.5) indicated that the attention was performed with high quality. When relating the sociodemographic variables with the quality level and of satisfaction perceived, there was no significant statistic association with any of the studied variables. Conclusion: the quality and global satisfaction levels in the dental services are perceived satisfactorily by the participants; however, this is not clear evidence of the existence of said quality, which forces the institution to follow up on the satisfaction of the users, since some indicators, such as the human resources department, include evidence of dissent.
\end{abstract}

Keywords: dental care, healthcare quality, medical-patient relationships, patient satisfaction.

\section{Percepção de satisfação e qualidade de serviços odontológicos em uma clínica universitária de Cartagena, Colômbia}

Resumo. Introdução: o escopo desta investigação foi descrever o nível de qualidade e satisfação percebido por usuários dos usuários dos serviços odontológicos de uma clínica universitária em Cartagena, Colômbia. Métodos: estudo de corte transversal em 277 pacientes. Para a coleta da informação, realizou-se um questionário autoministrado que continha variáveis demográficas, informação associada à prestação do serviço e questões especificas para qualidade e satisfação nos serviços. A análise da informação foi realizada com estatística descritiva a partir de frequências e percentagens, intervalos de confiança a $95 \%$ e análise bivariada onde foi utilizado o teste Chi-quadrado para estabelecer associação entre as variáveis. Resultados: mais da metade dos usuários dos serviços odontológicos foram mulheres; a idade média dos participantes foi de 39 anos (DE: 12,48); 99,3\% (IC $95 \%$ : 98,2-100) dos indivíduos indicou estar satisfeito com os serviços, e 97,8\% (IC 95\%: 96,1 $99,5)$ percebeu que o atendimento é prestado com qualidade. Relacionando as variáveis sócio-demográficas com o nível de qualidade e satisfação percebido, não se achou associação estatisticamente significativa com nenhuma das variáveis estudadas. Conclusão: o nível de qualidade e satisfação global nos serviços odontológicos são percebidos satisfatoriamente pelos usuários; no entanto, isto não é evidencia fidedigna da existência de tal qualidade, o que manda à instituição a fazer o acompanhamento da satisfação dos usuários, dado que em alguns indicadores como o recurso humano existe evidencia de desacordo.

Palavras chave: atendimento odontológico, qualidade do atendimento de saúde, relações médico-paciente, satisfação do paciente. 


\section{Introducción}

En la actualidad, dada la alta difusión de información y de estrategias de mercadeo en torno a la salud, es mayor el interés de la población hacia esta área y, por ende, es mayor la demanda de los servicios de salud. Los servicios odontológicos se convierten en un atractivo para los pacientes, quienes buscan satisfacer algunas necesidades funcionales y estéticas, debido a que la boca constituye un componente de salud que interviene en diversas funciones cotidianas indispensables para la persona, como la sonrisa, la alimentación y la fonación y, por lo tanto, facilita la comunicación [1].

A su vez, la prestación de servicios en salud requiere responder a ciertas expectativas de los usuarios y de los proveedores de salud. Para el caso de estos últimos, surgen indicadores para determinar cómo proporcionar el más alto nivel de calidad al menor costo, de la forma más equitativa posible y al mayor número de personas; sin embargo, es importante reconocer que la calidad es un concepto al que se le han atribuido múltiples definiciones, de modo que usuarios y proveedores lo perciben de diferentes maneras [2]. Esto se convierte en una necesidad para que las instituciones prestadoras de servicios en salud desarrollen investigaciones y seguimientos a sus procesos con el fin de evaluar la calidad de forma objetiva [3].

Así, en los últimos años, la perspectiva de los usuarios ha adquirido un auge como estrategia para la evaluación de la calidad de los servicios, utilizando el modelo de satisfacción del usuario con enfoque en el concepto de calidad de la atención propuesto por Donabedian [2] en 1966, pues se concibe como el tipo de atención que espera maximizar una medida integradora de bienestar del paciente después de considerar el saldo de las ganancias y las pérdidas esperadas asociadas con el proceso de atención en todas sus fases [4].

Los programas académicos de ciencias de la salud, como la Odontología, exigen la articulación entre la teoría y la práctica dentro del proceso de enseñanza-aprendizaje. El desarrollo de habilidades y competencias clínicas integrales permite solucionar las necesidades de cada paciente, pues cada uno representa una situación de salud única a partir de la cual el estudiante puede integrar y sintetizar el conocimiento científico adquirido [5].

Lasfacultades de Odontología pueden alcanzar este propósito por medio de los servicios docente- asistenciales, en los cuales el paciente es un elemento primordial para la práctica del estudiante, quien está en un proceso de aprendizaje para convertirse en profesional. Aunado a lo anterior, dada la naturaleza de estos servicios, durante los procedimientos se proporciona una doble atención que integra habilidades humanas y científicas tanto del docente como del estudiante, y las mismas condiciones de la academia llevan al seguimiento de protocolos y estándares quizá más exigentes que los empleados en otros servicios odontológicos, por lo que estas particularidades pueden influir en la satisfacción y la calidad percibidas por los pacientes.

De otra parte, los modelos de enseñanza-aprendizaje en Odontología, según Eriksen [6], deben estar centrados en el paciente, por lo cual uno de estos modelos propone los estándares de calidad más altos y otorga un reconocimiento de la mejor práctica hacia el paciente. Debido a esto, resulta relevante considerar la percepción de calidad y la satisfacción de los pacientes que asisten a este tipo de servicios asistenciales dentro de las escuelas de formación odontológica, con el fin de hacer un control y una evaluación de los servicios para identificar fortalezas y la necesidad de implementar modificaciones que garanticen los mejores resultados tanto en los pacientes como en el proceso de enseñanza-aprendizaje de docentes y estudiantes.

Teniendo en cuenta lo anterior, el objetivo del presente artículo es describir la percepción de calidad y satisfacción de los usuarios de los servicios odontológicos de una clínica universitaria en Cartagena.

\section{Materiales y métodos}

\section{Selección y descripción de los participantes}

Estudio descriptivo de corte transversal en 277 pacientes empleando un muestreo censal para los inscritos-activos en las clínicas integrales y a partir del cumplimiento de los criterios de selección. Se incluyeron adultos tratados por estudiantes de sexto a décimo semestre de la Escuela de Odontología de la Universidad del Sinú Elías Bechara Zainúm, seccional Cartagena, durante el primer periodo de 2013 y que voluntariamente participaron en la investigación. Se excluyeron aquellos 
que no tuvieran la historia clínica totalmente diligenciada, firmada y con el anexo de la cédula de ciudadanía.

La aplicación del instrumento para recolección de información consideró los aspectos éticos estipulados en la normatividad nacional vigente para investigación en salud, según la Resolución 8430, que estableció que el presente estudio corresponde a riesgo mínimo [7]. Además, se informó a los usuarios citados a la clínica odontológica sobre los beneficios que generaría la investigación, y sobre aspectos relacionados con la autonomía para pertenecer o no al estudio y con la confidencialidad de la información por medio del consentimiento informado.

\section{Información técnica}

\section{Instrumento para recolección de información}

Se diseñó una encuesta autosuministrada que incluyó datos de identificación e información sociodemográ- fica; además de ocho ítems (I1-I8) para obtener datos sobre satisfacción percibida con respecto a los servicios recibidos y que podrían ser resueltos, considerando una escala de Likert de 1 a 5, desde 1 (Completamente insatisfecho), 2 (Medianamente insatisfecho), 3 (Me da igual), 4 (Medianamente satisfecho) hasta 5 (Completamente satisfecho).

Adicional a esto, se incluyeron ocho preguntas (P9-P16) que correspondían a la calidad percibida en los servicios, cuya respuesta podía ser afirmativa o negativa (figura 1). Es preciso señalar que los ítems y las preguntas se agruparon considerando: (i) indicadores para satisfacción en los servicios, como recurso humano (I1, I3), infraestructura (I2, I4), bioseguridad (I6, I8), información (I5) y tiempo de tratamiento (I7); (ii) indicadores para calidad de los servicios: información (P9, P10, P12), bioseguridad (P11), efectividad (P13), eficacia (P14) y aceptabilidad (P15, P16). También, se incluyeron cuatro preguntas en referencia al costo y al pago de los tratamientos con el fin de obtener información complementaria.

Ítems (I1-I8) y preguntas (P9-P16)

I1. La forma como lo recibió la recepcionista de la clínica.

I2. a) Ambiente de la sala de espera.

b) Cantidad de tiempo esperado para recibir la atención.

I3. a) La forma como lo recibió el odontólogo.

b) La presentación personal del odontólogo que le atendió.

I4. La infraestructura, el estado de las instalaciones y los equipos de la unidad de trabajo del estudiante.

I5. La información del estado de su cavidad oral y del tratamiento a llevar a cabo le fue suministrada en términos que usted conociera.

I6. Siente usted que el odontólogo tenga confianza al momento de realizar el procedimiento.

I7. El tiempo de duración del tratamiento de acuerdo con el tipo de procedimiento realizado.

I8. a) La calidad del material.

b) El instrumental está limpio.

c) Organización del lugar de trabajo.

P9. ¿Firmó usted un consentimiento informado antes del procedimiento?

P10. ¿Firmó usted la constancia del tratamiento realizado en la historia clínica?

P11. ¿El estudiante, al momento de atenderlo, utilizó bata, gorro, tapabocas, guantes?

P12. ¿Le fue realizado todo el plan de tratamiento propuesto al inicio de su consulta?

P13. ¿Está satisfecho con el resultado final de su procedimiento?

P14. ¿Está satisfecho con el número de citas que debió cumplir para realizar su procedimiento?

P15. Después de recibir el servicio odontológico, ¿volvería a consulta a la Clínica Odontológica Cartagena de Indias?

P16. ¿Recomendaría la Clínica Odontológica Cartagena de Indias a otras personas para realizarse procedimientos odontológicos?

Figura 1. Ítems y preguntas contenidas en el cuestionario autosuministrado diseñado por los autores Fuente: elaboración propia 
El proceso de construcción y calibración del instrumento se llevó a cabo mediante el juicio de expertos en la temática, quienes verificaron la validez de apariencia logrando la suficiencia y pertinencia de las variables. Las preguntas se seleccionaron según su relevancia en el contexto y una previa revisión de la literatura [8]. Para lograr la estandarización de los auxiliares de investigación encargados de la recolección de la información y para determinar la comprensión de las preguntas, se hizo una prueba piloto en 20 pacientes adultos de la Clínica Odontológica Cartagena de Indias, quienes fueron excluidos de la muestra final del estudio. Después de esto, se hicieron las modificaciones necesarias para usar el instrumento con los usuarios que cumplieron con los criterios de inclusión.

\section{Estadística}

Una vez recolectada la información, se tabuló en una matriz de datos en Excel 2010 y luego fue transportada al software STATA ${ }^{\circledR}$ versión para Windows 11.0. Se procedió a hacer un análisis descriptivo por medio de frecuencias y porcentajes; para establecer asociación entre las variables, se utilizó la prueba Chi-cuadrado asumiendo un límite de significancia de 0,05 .

Al hacer el análisis de los ítems 1 a 8 (I1-I8), responder al menos cinco ítems con valor de puntuación 3, 4 o 5 se consideró como satisfacción; si el paciente respondió al menos cinco ítems con puntuación de 1 o 2, fue calificado como insatisfecho. En caso de los ítems I2, I3 e I8, que a su vez contenían dos o tres preguntas inmersas, se hizo un puntaje total promediado por ítem. Para las preguntas P9-P16, cuando hubo una respuesta afirmativa, se asignó una puntuación de 1 , en tanto que para la respuesta negativa, se asignó una puntuación de 0 . Se consideró la existencia de calidad cuando la sumatoria del puntaje de cada pregunta arroja un valor $\geq 5$; en caso contrario, se determinó ausencia de calidad.

Las preguntas relacionadas con el costo y el pago de los tratamientos fueron específicas para cierta situación, y los participantes solo tuvieron dos opciones de respuesta (afirmativa y negativa).

\section{Resultados}

Con referencia a las características sociodemográficas, se puede destacar que la edad promedio fue de 39 años (DE: 12,48). Más de la mitad de los sujetos del estudio corresponde al sexo femenino y está en estrato medio. Cerca del $50 \%$ de los pacientes está distribuido entre sexto y séptimo semestre para recibir los servicios de atención en salud bucal (tabla 1).

Tabla 1. Variables sociodemográficas de los sujetos de estudio

\begin{tabular}{|l|c|c|}
\hline $\begin{array}{c}\text { Variables sociodemo- } \\
\text { gráficas }\end{array}$ & $\begin{array}{c}\text { Frecuencia } \\
\mathbf{n = 2 7 7}\end{array}$ & $\%$ \\
\hline Edad & 129 & 46,6 \\
\hline 18 a 37 años & 126 & 45,5 \\
\hline 38 a 57 años & 22 & 7,9 \\
\hline 58 a 78 años & 175 & 63,2 \\
\hline Sexo & 102 & 36,8 \\
\hline Femenino & & \\
\hline Masculino & 94 & 33,9 \\
\hline Estrato socioeconómico & 178 & 64,2 \\
\hline Bajo & 5 & 1,8 \\
\hline Medio & 69 & 24,9 \\
\hline Alto & 69 & 24,9 \\
\hline $\begin{array}{l}\text { Semestre en el que recibe } \\
\text { servicios }\end{array}$ & 62,4 \\
\hline Sexto & & 17 \\
\hline Séptimo & & \\
\hline Octavo & & \\
\hline Noveno & & \\
\hline Décimo & & \\
\hline
\end{tabular}

Fuente: elaboración propia

\section{Análisis global para satisfacción y calidad}

En el análisis global, se encontró que el 99,3\% (IC 95\%: 98,2-100) de los sujetos manifestó estar satisfecho con los servicios prestados por la institución, considerando los indicadores para satisfacción en los servicios, como recurso humano (I1, I3), infraestructura (I2, I4), bioseguridad (I6, I8), 
información (I5) y tiempo de tratamiento (I7); a su vez, 97,8\% (IC 95\%: 96,1-99,5) percibió que la atención se presta con calidad al considerar los indicadores de calidad de los servicios, representados por: información (P9, P10, P12), bioseguridad (P11), efectividad (P13), eficacia (P14) y aceptabilidad (P15, P16) (tabla 2).

Tabla 2. Análisis global de satisfacción y calidad percibida en los servicios por sujetos de estudio

\begin{tabular}{|l|l|l|l|}
\hline \multicolumn{1}{|c|}{$\begin{array}{c}\text { Dimensión } \\
\text { global }\end{array}$} & Frecuencia & \multicolumn{1}{c|}{ IC 95\% } & \\
\hline Satisfacción & & & \\
\hline Insatisfecho & 2 & 0,7 & $-0,2-1,7$ \\
\hline Satisfecho & 275 & 99,3 & $98,2-100$ \\
\hline Calidad & & & \\
\hline No hay calidad & 6 & 2,2 & $0,4-3,8$ \\
\hline Sí hay calidad & 271 & 97,8 & $96,1-99,5$ \\
\hline
\end{tabular}

Fuente: elaboración propia

\section{Indicadores de satisfacción}

En referencia a la satisfacción percibida en usuarios de los servicios de la clínica odontológica, es posible mencionar que al indagar por recurso humano, infraestructura, bioseguridad, información y tiempo de tratamiento, 98,9\% (IC 95\%: 97,6-100) de los sujetos se mostró satisfecho con los indicadores de bioseguridad y de información en $96 \%$ (IC $95 \%$ : 93,7-98,3), y cerca del $80 \%$ de los encuestados manifestó estar satisfecho con la atención recibida por el recurso humano (tabla 3 ).

\section{Indicadores de calidad}

La mayoría de los sujetos del estudio percibió la existencia de calidad para la información, la bioseguridad, la efectividad y la aceptabilidad, en tanto que el 21,3\% (IC 95\%: 16,4-26,1) evidenció considerar ausencia de calidad en lo que se refiere a eficacia en los procedimientos, lo cual está representado por la respuesta negativa ante la pregunta P14 (está satisfecho con el número de citas que debió cumplir para la realización de sus procedimientos; véase tabla 3 ).
Tabla 3. Distribución para indicadores de satisfacción y calidad percibidas por los usuarios

\begin{tabular}{|c|c|c|c|}
\hline \multicolumn{4}{|c|}{ Indicadores de satisfacción } \\
\hline & Frecuencia & $\%$ & IC $95 \%$ \\
\hline \multicolumn{4}{|c|}{ Recurso humano } \\
\hline Insatisfecho & 58 & 20 & $16,1-25,7$ \\
\hline Satisfecho & 219 & 80 & $74,2-83,8$ \\
\hline \multicolumn{4}{|c|}{ Infraestructura } \\
\hline Insatisfecho & 39 & 14,08 & $9,9-18,2$ \\
\hline Satisfecho & 238 & 85,92 & $81,7-90$ \\
\hline \multicolumn{4}{|l|}{ Bioseguridad } \\
\hline Insatisfecho & 3 & 1,1 & $-0,1-2,3$ \\
\hline Satisfecho & 274 & 98,9 & $97,6-100$ \\
\hline \multicolumn{4}{|l|}{ Información } \\
\hline Insatisfecho & 11 & 4 & $1,6-6,2$ \\
\hline Satisfecho & 266 & 96 & $93,7-98,3$ \\
\hline \multicolumn{4}{|c|}{ Tiempo de tratamiento } \\
\hline Insatisfecho & 25 & 9 & $5,6-12,4$ \\
\hline Satisfecho & 252 & 91 & $87,5-94,3$ \\
\hline \multicolumn{4}{|c|}{ Indicadores de calidad } \\
\hline \multicolumn{4}{|l|}{ Información } \\
\hline No & 7 & 2,5 & $0,6-4,3$ \\
\hline Sí & 270 & 97,5 & $95,6-99,3$ \\
\hline \multicolumn{4}{|c|}{ Bioseguridad } \\
\hline No & 6 & 2,2 & $0,4-3,8$ \\
\hline Sí & 271 & 97,8 & $96,1-99,5$ \\
\hline \multicolumn{4}{|l|}{ Efectividad } \\
\hline No & 13 & 4,7 & $2,1-7,1$ \\
\hline Sí & 264 & 95,3 & $92,8-97,8$ \\
\hline \multicolumn{4}{|l|}{ Eficacia } \\
\hline No & 59 & 21,3 & $16,4-26,1$ \\
\hline Sí & 218 & 78,7 & $73,8-83,5$ \\
\hline \multicolumn{4}{|c|}{ Aceptabilidad } \\
\hline No & 5 & 1,8 & $0,2-3,3$ \\
\hline Sí & 272 & 98,2 & $96,6-99,7$ \\
\hline
\end{tabular}

Fuente: elaboración propia

\section{Costo y pago de los tratamientos}

Al indagar por el costo y el pago de los tratamientos, cerca del $91 \%$ de los pacientes encuestados aceptó que el estudiante es quien hace el pago por el tra- 
tamiento recibido; sin embargo, en otro de los interrogantes se reporta que en más de la mitad de los casos el pago es distribuido entre pacientes y estudiantes. Respecto al costo pagado, cerca del $89 \%$ de los pacientes se mostraba satisfecho (tabla 4).

Tabla 4. Costos y pagos de los tratamientos

\begin{tabular}{|l|c|c|c|}
\hline \multicolumn{1}{|c|}{ Pregunta } & Frecuencia & Porcentaje & IC 95\% \\
\hline $\begin{array}{l}\text { ¿El estudiante pagó } \\
\text { por usted el trata- } \\
\text { miento recibido? }\end{array}$ & & & \\
\hline No & 23 & 8,30 & $5,0-11,5$ \\
\hline Sí & 254 & 91,70 & $88,4-94,9$ \\
\hline $\begin{array}{l}\text { ¿'Usted pagó el 50\% } \\
\text { del tratamiento reci- } \\
\text { bido y el estudiante } \\
\text { el otro 50\%? }\end{array}$ & & & \\
\hline No & 6 & 33,33 & $9,2-57,4$ \\
\hline Sí & 12 & 66,67 & $42,5-90,7$ \\
\hline $\begin{array}{l}\text { ¿Pagó en su totali- } \\
\text { dad el tratamiento } \\
\text { recibido? }\end{array}$ & & & \\
\hline No & 12 & 66,67 & $42,5-90,7$ \\
\hline Sí & 6 & 33,33 & $9,2-57,4$ \\
\hline $\begin{array}{l}\text { ¿Le pareció } \\
\text { adecuado el valor } \\
\text { cancelado? }\end{array}$ & 16 & 11,11 & $-4,97-27,1$ \\
\hline No & & 88,89 & $72,8-100$ \\
\hline Sí & & & \\
\hline
\end{tabular}

Fuente: elaboración propia

\section{Análisis bivariado}

El análisis bivariado mostró que al relacionar la satisfacción y la calidad percibidas en los pacientes usuarios de los servicios de la clínica odontológica con variables demográficas como edad, sexo y estrato, no se encontraron relaciones estadísticamente significativas $(\mathrm{P}>0,05)$.

\section{Discusión}

Una limitación del presente estudio es que en las clínicas odontológicas de instituciones universitarias, a diferencia de las clínicas odontológicas de prestadores públicos y privados, hay profesores (clínicos especializados) que supervisan la prestación de los servicios. Esto es un elemento diferencial con respecto a la atención que se brinda, dado que posibilita que los pacientes reciban doble atención (tanto del profesor que tiene las competencias disciplinares específicas en el área de atención requerida como del estudiante de odontología que está en proceso de formación académica para el desarrollo de sus competencias), lo que puede influir en la satisfacción y en la calidad percibidas.

De otra parte, es preciso mencionar que otro posible factor de relevancia para comprender los resultados de calidad y satisfacción percibidas es el que concierne al costo y el pago de los tratamientos recibidos. Se evidenció que muchos de los pagos por concepto de tratamientos recibidos no son asumidos únicamente por los pacientes, puesto que los estudiantes contribuyen hasta en un $50 \%$ con ellos y en ocasiones los asumen en su totalidad, hecho que sin duda es un valor agregado en la atención y que los pacientes podrían llegar a interpretar como un factor adicional para calificar la calidad en los servicios y generar mayor satisfacción.

Respecto al hecho de que los estudiantes paguen los tratamientos, se genera cierta preocupación sobre su economía, pues deben adicionar este gasto a su inversión en matrícula, materiales y gastos personales, entre otros. Además, dicha situación es inquietante al requerir la comprensión de por qué los estudiantes deben asumir los costos de servicios que ellos mismos brindan. No obstante, es pertinente señalar que responder a tales interrogantes no forma parte del propósito de esta investigación, por lo cual se sugiere para próximos estudios incluir ítems y preguntas en el instrumento que permitan acceder a dichos motivos.

En el presente estudio, se obtuvo una aproximación del grado de satisfacción de los usuarios acerca de los servicios de una clínica odontológica universitaria de Cartagena. Esto resulta importante para la institución y el recurso humano (docentes y estudiantes), dado que la satisfacción del paciente es considerada un indicador deseable de la prestación de un servicio y debe ser uno de los objetivos de los prestadores del servicio [9].

Es relevante destacar que si el usuario es el demandante del servicio y quien mejor conoce sus propias necesidades, expectativas y aprensiones como "consumidor" [10], la mejor forma de obtener una aproximación de su grado de satisfacción es involucrándolo para conocer su percepción; así se hizo en esta investigación, en la que la mayoría de usuarios estaba satisfecho con los servicios recibidos. Esto probablemente contribuye a reducir 
actitudes negativas que afectan la aceptación del tratamiento y que dificultan la prestación del servicio por estudiantes y docentes, para el caso de una clínica odontológica universitaria; este hecho impactaría de forma negativa no solo el bienestar del paciente, sino también los procesos de formación académica del estudiante y la labor docente desarrollada.

La mayoría de los usuarios reportó satisfacción y conformidad respecto a ciertos indicadores como información y bioseguridad; para este último indicador, que incluye la percepción sobre la calidad del material, la higiene del instrumental y la organización del lugar de trabajo, solo $1 \%$ de los individuos se mostró insatisfecho, resultado que concuerda con los reportes hechos por Elizondo en el 2011 [11] sobre una clínica odontológica universitaria en México, pues la variable de seguridad percibida por el paciente se ubicó mayoritariamente en las escalas de muy satisfactorio y satisfactorio.

El alto grado de satisfacción de los usuarios respecto al servicio recibido está relacionado con el recurso humano, sobre todo con el trato amable, tal como lo reporta un estudio con usuarios de servicios dentales públicos dirigidos a población de bajos ingresos en México D. F. [12]. Pese a que en el presente estudio tan solo un $20 \%$ de los sujetos estuvo inconforme con el recurso humano, fue el dominio con mayor valor de inconformidad, ya sea por la forma en que lo recibió el recepcionista y el odontólogo o por la presentación personal de quien le atendió.

La anterior situación reviste importancia, pues denota cierta limitación del recurso humano en salud para desempeñar sus funciones y brindar una atención en salud de calidad. Este hecho está relacionado con la actual crisis de recursos humanos en salud, representada no solo por la reducción en el número de individuos prestadores del servicio, sino también por la disminución de habilidades propias y competencias sociales [13], entre las cuales se puede mencionar la comunicación y el trato a los semejantes.

A pesar de la existencia de particularidades antes mencionadas para ciertos indicadores, el análisis global mostró que la mayoría de los sujetos está satisfecho con los servicios recibidos y estos resultados son similares a los reportados en Colombia por López en el 2010 [9] con un $83 \%$ de satisfacción en los usuarios del servicio y por Hincapié en el 2004 [14] con 95,6\%.
Lo anterior puede deberse a que los servicios son de calidad o a que en razón a una condición socioeconómica similar entre los habitantes de un país en vía de desarrollo como Colombia, los pacientes tienen percepciones, necesidades, expectativas y demandas de los servicios muy parecidas. Sin embargo, al comparar estos resultados con los de usuarios en un país socioeconómico y culturalmente diferente como Estados Unidos, el reporte de Bedi en 2005 [15] revela que solo el $2 \%$ de los usuarios se ha quejado de los servicios odontológicos, lo que sugiere que en Estados Unidos es favorable la percepción de los usuarios. Aunque la satisfacción antes reportada podría reflejar solo actitudes como "ellos hacen lo mejor que pueden" o "mi satisfacción no es realmente su trabajo" [16], por lo cual hay unos factores intervinientes cuyo control no fue considerado en esta investigación y puede generar sesgo.

Para la presente investigación, casi la totalidad de los usuarios manifestó recibir una atención con calidad en dominios como la información, la bioseguridad, la efectividad, la aceptabilidad y la eficacia. Resultados muy similares son los reportados por Betin en el 2009 [17], en un estudio realizado en Cartagena, en el que casi el $90 \%$ de los usuarios de una clínica odontológica universitaria pública manifestó estar satisfecho con la calidad de la atención recibida.

Para el dominio eficacia, representado por la pregunta 14 (que indaga si hay satisfacción con el número de citas que debió cumplir para llevar a cabo los procedimientos), la conformidad de la mayoría de los usuarios denota una buena distribución del tiempo durante la prestación de los servicios, pese a que se conoce que los tratamientos pueden tardar un poco más en los servicios odontológicos universitarios debido a la realización paralela de actividades académicas con los estudiantes.

La situación de satisfacción con el número de citas también pudo haber influido en dominios como el de información, dado que algunos usuarios por ejemplo consideran que la información recibida sobre diagnósticos y tratamientos se percibe de manera más favorable en relación con el número de citas, tal como lo señala un estudio de Alfaro realizado en Perú (2011) [18].

Es importante ser estricto con el número de citas para efectuar adecuadamente los procedimientos, pues acudir a citas excesivas para culminar los tratamientos puede generar una sensación 
de falta de calidad y esto ha sido calificado como un motivo muy frecuente en la deserción de procedimientos odontológicos [19]; en el caso de una clínica odontológica universitaria esto no solo afectaría el éxito de los tratamientos, sino también el curso académico de los estudiantes.

Se ha descrito que las actitudes y los sentimientos positivos hacia los servicios se forman ya sea como resultado de la propia interacción dentista-paciente o por recomendación de otros individuos, generalmente familiares o amigos, de acuerdo con sus experiencias [20]. La respuesta afirmativa y satisfactoria en la mayoría de los casos de este estudio para el dominio de aceptabilidad (que incluye la pregunta de si volvería a consulta en la Clínica Odontológica Cartagena de Indias y si recomendaría los servicios de la clínica) genera la posibilidad de que otros usuarios lleguen al servicio por la recomendación de los usuarios encuestados.

Cada vez es mayor la participación de los usuarios en la demanda de servicios odontológicos. Por lo tanto, los determinantes de calidad que se enfocan en los usuarios [21] han tomado mucho auge, dado que es mejor obtener información de percepción de calidad directamente de quien recibe el servicio (usuario) para hacer las modificaciones acorde a las necesidades reales, por lo cual el presente estudio consideró un análisis de calidad global que mostró, en la mayoría de los usuarios, una respuesta afirmativa.

A pesar de que existe un modelo de conformidad, satisfacción y calidad de los servicios recibidos en una clínica odontológica universitaria de Cartagena, es necesario el desarrollo de estudios longitudinales para identificar las variaciones en la percepción de satisfacción y calidad de los servicios y para hacer estudios específicos sobre el dominio de recurso humano (constituido por profesores y estudiantes, quienes brindan atención conjunta en las clínicas universitarias), pues hubo una leve inconformidad de los usuarios.

Asimismo, es imperativo indagar e identificar las posibles causas que inducen a la insatisfacción percibida, y si esta deriva de la atención conjunta entre profesor y estudiante o si quizá favorece a alguno de los dos, con el propósito de hacer las intervenciones oportunas para mejorar la prestación de los servicios y contribuir al óptimo desempeño de profesores y estudiantes.

\section{Agradecimientos}

A Yenifer Batista Mendoza, Yandry Milett Blanco Castellón y Nilson Ortiz Zárate, por su valioso apoyo en el proceso de recolección de información.

\section{Referencias}

[1] Bennadi D, Reddy C. Oral health related quality of life. J Int Soc Prevent Communit Dent. 2013;3(1):1-6.

[2] Delgado ME, Vásquez ML, De Moraes L. Calidad en los servicios de salud desde los marcos de sentido de diferentes actores sociales en Colombia y Brasil. Rev Salud Pública. 2010;12(4):533-45.

[3] Navarrete S, Gómez A, Riebeling C, López G, Nava A. La investigación sobre calidad de la atención en el Instituto Mexicano del Seguro Social. Estudio bibliométrico. Rev Salud Pública México. 2013;55(6):564-71.

[4] Mittermayer R, Huic M, Mestrovic J. Quality of health care, accreditation, and health technology assessment in Croatia: role of agency for quality and accreditation in health. Acta Med Croatica. 2010;64(5):425-34.

[5] Álvarez Loureiro L, Gugelmeier V, Hermida Bruno L. Cómo aprenden los estudiantes de odontología que cursan el último año de la carrera. Odontoestomatología. 2013;15(21):4-11.

[6] Rangel Galvis MC. Perspectiva bioética y modelo biopsicosocial en la relación odontólogo-paciente durante la formación del estudiante en la Universidad del Bosque. Revista Salud Bosque. 2011;1(2):87-98.

[7] Colombia. Ministerio de Salud. Resolución 8430, Por la cual se establecen las normas científicas, técnicas y administrativas para la investigación en salud. Bogotá: Ministerio de Salud. Disponible en: https:// www.invima.gov.co/images/pdf/medicamentos/resoluciones/etica_res_8430_1993.pdf

[8] Martín MC. Diseño y validación de cuestionarios. Matronas Profesión. 2004;5:23-9.

[9] López O, Cerezo M, Paz A. Variables relacionadas con la satisfacción del paciente de los servicios odontológicos. Rev Gerenc Polit Salud. 2010;9(18):124-36.

[10] Araya Vallespir C, Bustos Leal A, Castillo F, Oliva Belmar P, Araya Gozalvo J. Determinación de la calidad del servicio odontológico de un centro de salud pública basado en intangibles: Talcahuano, Chile. Int J Odontostomat. 2012;6(3):349-54.

[11] Elizondo J, Quiroga MA, Palomares P, Martínez G. La satisfacción del paciente con la atención técnica 
del servicio odontológico. Revista Salud Pública y Nutrición. 2011 Enero-marzo;12(1). Disponible en: http://www.medigraphic.com/pdfs/revsalpubnut/ spn-2011/spn111d.pdf

[12] Lara N, López CV, Morales S. Satisfacción de usuarios de dos servicios odontológicos en Tláhuac, Distrito Federal. Revista ADM. 2000;57(2):45-9.

[13] Soto A. Recursos humanos en salud. Rev Perú Med Exp Salud Pública. 2011;28(2):173-4.

[14] Hincapié RA, Carvajal FA, Sánchez LA, Arias RO. Satisfacción de los usuarios frente a la calidad de la atención prestada en el programa de odontología. Rev Fac Odontol Univ Antioq. 2004;15(2):5-11.

[15] Bedi R, Gulati N, McGrath C. A study of satisfaction with dental services among adults in the United Kingdom. Brit Dent J. 2005;198:433-7.

[16] Newsome PRH, Wright GH. Qualitative techniques to investigate how patients evaluate dentists: a pilot study. Community Dent Oral Epidemiol. 2000;28(4):257-66.

[17] Betin A. Guardela A, Infante K, Díaz A, González M. Satisfacción de los pacientes respecto a la calidad de la atención odontológica en una institución pública universitaria de la ciudad de Cartagena. Duazary Med. 2009;6(2):95-101.

[18] Alfaro-Carballido LD, García-Rupaya CR. Percepción del proceso de consentimiento informado en pacientes de la Clínica Estomatológica Central de la Universidad Peruana Cayetano Heredia. Rev Estomatol Herediana. 2011;21(1):5-12.

[19] Bucchi C, Sepúlveda, C, Monsalve M, Bustos L. Descripción de la satisfacción usuaria de pacientes que reciben atención de urgencia dental en cinco establecimientos de atención primaria de salud. Int J Odontostomat. 2012;6(3):275-80.

[20] Lara N, López V. Factores que influyen en la utilización de los servicios odontológicos. Revisión de la literatura. Revista ADM. 2002;59(3):100-9.

[21] Agudelo AA, Valencia LY, Oullón E, Betancur LG, Restrepo LM, Peláez LV. Satisfacción y factores asociados en estudiantes usuarios del servicio odontológico de la institución prestadora de servicios de salud IPS Universitaria (Medellín). Rev Fac Odontol Univ Antioq. 2008;19(2):13-23. 